\title{
Niemann-Pick Disease
}

National Institute of Neurological Disorders and Stroke (NINDS)

\section{Source}

National Institute of Neurological Disorders and Stroke (NINDS). Niemann-Pick Disease Information Page.

Niemann-Pick disease (NP) refers to a group of inherited metabolic disorders known as lipid storage diseases. Lipids (fatty materials such as waxes, fatty acids, oils, and cholesterol) and proteins are usually broken down into smaller components to provide energy for the body. In Niemann-Pick disease, harmful quantities of lipids accumulate in the brain, spleen, liver, lungs, and bone marrow. Neurological symptoms may include ataxia (lack of muscle control during voluntary movements such as walking), loss of muscle tone, brain degeneration, increased sensitivity to touch, spasticity (stiff muscles and awkward movement), and slurred speech. Other symptoms may include feeding and swallowing difficulties, eye paralysis, learning problems, and an enlarged liver and spleen. There may be clouding of the cornea and a characteristic cherry-red halo develops around the center of the retina.

The disease has three categories.

- Type A, the most severe form, begins in early infancy and occurs most often in Jewish families. Additional symptoms include weakness, an enlarged liver and spleen, swollen lymph nodes, and profound brain damage by six months of age. Children with this type rarely live beyond 18 months.

- Type B (called juvenile onset) usually occurs in the pre-teen years, with symptoms that include ataxia and peripheral neuropathy. The brain is generally not affected. Other symptoms include enlarged liver and spleen, and pulmonary difficulties. In types $\mathbf{A}$ and $\mathbf{B}$, insufficient activity of an enzyme called sphingomyelinase causes the build up of toxic amounts of sphingomyelin, a fatty substance present in every cell of the body.

- Type C may appear early in life or develop in the teen or adult years. It is caused by a lack of the NPC1 or NPC2 proteins. Affected individuals may have extensive brain damage that can cause an inability to look up and down, difficulty in walking and swallowing, and progressive loss of vision and hearing. There may be moderate 
enlargement of the spleen and liver. 